\title{
Stability of a Generalized Euler-Lagrange Type Additive Mapping and Homomorphisms in $C^{*}$-Algebras
}

\author{
Abbas Najati ${ }^{1}$ and Choonkil Park ${ }^{2}$ \\ ${ }^{1}$ Department of Mathematics, Faculty of Sciences, University of Mohaghegh Ardabili, \\ Ardabil, 56199-11367, Iran \\ ${ }^{2}$ Department of Mathematics, Research Institute for Natural Sciences, Hanyang University, \\ Seoul, 133-791, South Korea \\ Correspondence should be addressed to Choonkil Park, baak@hanyang.ac.kr \\ Received 17 June 2009; Revised 30 July 2009; Accepted 4 August 2009 \\ Recommended by Patricia J. Y. Wong
}

Let $X, Y$ be Banach modules over a $C^{*}$-algebra and let $r_{1}, \ldots, r_{n} \in \mathbb{R}$ be given. We prove the generalized Hyers-Ulam stability of the following functional equation in Banach modules over a unital $C^{*}$-algebra: $\sum_{j=1}^{n} f\left(-r_{j} x_{j}+\sum_{1 \leq i \leq n, i \neq j} r_{i} x_{i}\right)+2 \sum_{i=1}^{n} r_{i} f\left(x_{i}\right)=n f\left(\sum_{i=1}^{n} r_{i} x_{i}\right)$. We show that if $\sum_{i=1}^{n} r_{i} \neq 0, r_{i}, r_{j} \neq 0$ for some $1 \leq i<j \leq n$ and a mapping $f: X \rightarrow Y$ satisfies the functional equation mentioned above then the mapping $f: X \rightarrow Y$ is Cauchy additive. As an application, we investigate homomorphisms in unital $C^{*}$-algebras.

Copyright (c) 2009 A. Najati and C. Park. This is an open access article distributed under the Creative Commons Attribution License, which permits unrestricted use, distribution, and reproduction in any medium, provided the original work is properly cited.

\section{Introduction and Preliminaries}

The stability problem of functional equations originated from a question of Ulam [1] concerning the stability of group homomorphisms. Hyers [2] gave a first affirmative partial answer to the question of Ulam for Banach spaces. Hyers' theorem was generalized by Aoki [3] for additive mappings and by Th. M. Rassias [4] for linear mappings by considering an unbounded Cauchy difference.

Theorem 1.1 (Th. M. Rassias [4]). Let $f: E \rightarrow E^{\prime}$ be a mapping from a normed vector space $E$ into a Banach space $E^{\prime}$ subject to the inequality

$$
\|f(x+y)-f(x)-f(y)\| \leq \epsilon\left(\|x\|^{p}+\|y\|^{p}\right)
$$


for all $x, y \in E$, where $\epsilon$ and $p$ are constants with $\epsilon>0$ and $p<1$. Then the limit

$$
L(x)=\lim _{n \rightarrow \infty} \frac{f\left(2^{n} x\right)}{2^{n}}
$$

exists for all $x \in E$ and $L: E \rightarrow E^{\prime}$ is the unique additive mapping which satisfies

$$
\|f(x)-L(x)\| \leq \frac{2 \epsilon}{2-2^{p}}\|x\|^{p}
$$

for all $x \in E$. If $p<0$, then (1.1) holds for $x, y \neq 0$ and (1.3) for $x \neq 0$. Also, if for each $x \in E$ the mapping $t \mapsto f(t x)$ is continuous in $t \in \mathbb{R}$, then $L$ is $\mathbb{R}$-linear.

Theorem 1.2 (J. M. Rassias [5-7]). Let $X$ be a real normed linear space and $Y$ a real Banach space. Assume that $f: X \rightarrow Y$ is a mapping for which there exist constants $\theta \geq 0$ and $p, q \in \mathbb{R}$ such that $r=p+q \neq 1$ and $f$ satisfies the functional inequality

$$
\|f(x+y)-f(x)-f(y)\| \leq \theta\|x\|^{p}\|y\|^{q}
$$

for all $x, y \in X$. Then there exists a unique additive mapping $L: X \rightarrow Y$ satisfying

$$
\|f(x)-L(x)\| \leq \frac{\theta}{\left|2^{r}-2\right|}\|x\|^{r}
$$

for all $x \in X$. If, in addition, $f: X \rightarrow Y$ is a mapping such that the transformation $t \rightarrow f(t x)$ is continuous in $t \in \mathbb{R}$ for each fixed $x \in X$, then $L$ is linear.

The paper of Th. M. Rassias [4] has provided a lot of influence in the development of what we call the generalized Hyers-Ulam stability of functional equations. In 1994, a generalization of Theorems 1.1 and 1.2 was obtained by Găvruţa [8], who replaced the bounds $\varepsilon\left(\|x\|^{p}+\|y\|^{p}\right)$ and $\theta\|x\|^{p}\|y\|^{q}$ by a general control function $\varphi(x, y)$.

The functional equation

$$
f(x+y)+f(x-y)=2 f(x)+2 f(y)
$$

is called a quadratic functional equation. In particular, every solution of the quadratic functional equation is said to be a quadratic mapping. The generalized Hyers-Ulam stability problem for the quadratic functional equation was proved by Skof [9] for mappings $f: X \rightarrow Y$, where $X$ is a normed space and $Y$ is a Banach space. Cholewa [10] noticed that the theorem of Skof is still true if the relevant domain $X$ is replaced by an Abelian group. Czerwik [11] proved the generalized Hyers-Ulam stability of the quadratic functional equation. J. M. Rassias [12, 13] introduced and investigated the stability problem of Ulam for the Euler-Lagrange quadratic mappings (1.6) and

$$
f\left(a_{1} x_{1}+a_{2} x_{2}\right)+f\left(a_{2} x_{1}-a_{1} x_{2}\right)=\left(a_{1}^{2}+a_{2}^{2}\right)\left[f\left(x_{1}\right)+f\left(x_{2}\right)\right]
$$


Grabiec [14] has generalized these results mentioned above. In addition, J. M. Rassias [15] generalized the Euler-Lagrange quadratic mapping (1.7) and investigated its stability problem. Thus these Euler-Lagrange type equations (mappings) are called as EulerLagrange-Rassias functional equations (mappings).

The stability problems of several functional equations have been extensively investigated by a number of authors and there are many interesting results concerning this problem (see $[4-8,12,13,15-55])$.

Recently, C. Park and J. Park [45] introduced and investigated the following additive functional equation of Euler-Lagrange type:

$$
\begin{array}{r}
\sum_{i=1}^{n} r_{i} L\left(\sum_{j=1}^{n} r_{j}\left(x_{i}-x_{j}\right)\right)+\left(\sum_{i=1}^{n} r_{i}\right) L\left(\sum_{i=1}^{n} r_{i} x_{i}\right) \\
=\left(\sum_{i=1}^{n} r_{i}\right) \sum_{i=1}^{n} r_{i} L\left(x_{i}\right), \quad r_{1}, \ldots, r_{n} \in(0, \infty)
\end{array}
$$

whose solution is said to be a generalized additive mapping of Euler-Lagrange type.

In this paper, we introduce the following additive functional equation of EulerLagrange type which is somewhat different from (1.8):

$$
\sum_{j=1}^{n} f\left(-r_{j} x_{j}+\sum_{1 \leq i \leq n, i \neq j} r_{i} x_{i}\right)+2 \sum_{i=1}^{n} r_{i} f\left(x_{i}\right)=n f\left(\sum_{i=1}^{n} r_{i} x_{i}\right)
$$

where $r_{1}, \ldots, r_{n} \in \mathbb{R}$. Every solution of the functional equation (1.9) is said to be a generalized Euler-Lagrange type additive mapping.

We investigate the generalized Hyers-Ulam stability of the functional equation (1.9) in Banach modules over a $C^{*}$-algebra. These results are applied to investigate $C^{*}$-algebra homomorphisms in unital $C^{*}$-algebras.

Throughout this paper, assume that $A$ is a unital $C^{*}$-algebra with norm $\|\cdot\|_{A}$ and unit $e$, that $B$ is a unital $C^{*}$-algebra with norm $\|\cdot\|_{B}$, and that $X$ and $Y$ are left Banach modules over a unital $C^{*}$-algebra $A$ with norms $\|\cdot\|_{X}$ and $\|\cdot\|_{Y}$, respectively. Let $U(A)$ be the group of unitary elements in $A$ and let $r_{1}, \ldots, r_{n} \in \mathbb{R}$. For a given mapping $f: X \rightarrow Y, u \in U(A)$ and a given $\mu \in \mathbb{C}$, we define $D_{u, r_{1}, \ldots, r_{n}} f$ and $D_{\mu, r_{1}, \ldots, r_{n}} f: X^{n} \rightarrow Y$ by

$$
\begin{aligned}
& D_{u, r_{1}, \ldots, r_{n}} f\left(x_{1}, \ldots, x_{n}\right):=\sum_{j=1}^{n} f\left(-r_{j} u x_{j}+\sum_{1 \leq i \leq n, i \neq j} r_{i} u x_{i}\right)+2 \sum_{i=1}^{n} r_{i} u f\left(x_{i}\right)-n f\left(\sum_{i=1}^{n} r_{i} u x_{i}\right), \\
& D_{\mu, r_{1}, \ldots, r_{n}} f\left(x_{1}, \ldots, x_{n}\right):=\sum_{j=1}^{n} f\left(-\mu r_{j} x_{j}+\sum_{1 \leq i \leq n, i \neq j} \mu r_{i} x_{i}\right)+2 \sum_{i=1}^{n} \mu r_{i} f\left(x_{i}\right)-n f\left(\sum_{i=1}^{n} \mu r_{i} x_{i}\right)
\end{aligned}
$$

for all $x_{1}, \ldots, x_{n} \in X$. 


\section{Generalized Hyers-Ulam Stability of the Functional Equation (1.9) in Banach Modules Over a $C^{*}$-Algebra}

Lemma 2.1. Let $x$ and $y$ be linear spaces and let $r_{1}, \ldots, r_{n}$ be real numbers with $\sum_{k=1}^{n} r_{k} \neq 0$ and $r_{i}, r_{j} \neq 0$ for some $1 \leq i<j \leq n$. Assume that a mapping $L: x \rightarrow y$ satisfies the functional equation (1.9) for all $x_{1}, \ldots, x_{n} \in X$. Then the mapping $L$ is Cauchy additive. Moreover, $L\left(r_{k} x\right)=r_{k} L(x)$ for all $x \in \mathcal{X}$ and all $1 \leq k \leq n$.

Proof. Since $\sum_{k=1}^{n} r_{k} \neq 0$, putting $x_{1}=\cdots=x_{n}=0$ in (1.9), we get $L(0)=0$. Without loss of generality, we may assume that $r_{1}, r_{2} \neq 0$. Letting $x_{3}=\cdots=x_{n}=0$ in (1.9), we get

$$
L\left(-r_{1} x_{1}+r_{2} x_{2}\right)+L\left(r_{1} x_{1}-r_{2} x_{2}\right)+2 r_{1} L\left(x_{1}\right)+2 r_{2} L\left(x_{2}\right)=2 L\left(r_{1} x_{1}+r_{2} x_{2}\right)
$$

for all $x_{1}, x_{2} \in \chi x$. Letting $x_{2}=0$ in (2.1), we get

$$
2 r_{1} L\left(x_{1}\right)=L\left(r_{1} x_{1}\right)-L\left(-r_{1} x_{1}\right)
$$

for all $x_{1} \in \mathcal{X}$. Similarly, by putting $x_{1}=0$ in (2.1), we get

$$
2 r_{2} L\left(x_{2}\right)=L\left(r_{2} x_{2}\right)-L\left(-r_{2} x_{2}\right)
$$

for all $x_{1} \in \mathcal{X}$. It follows from (2.1), (2.2) and (2.3) that

$$
L\left(-r_{1} x_{1}+r_{2} x_{2}\right)+L\left(r_{1} x_{1}-r_{2} x_{2}\right)+L\left(r_{1} x_{1}\right)+L\left(r_{2} x_{2}\right)-L\left(-r_{1} x_{1}\right)-L\left(-r_{2} x_{2}\right)=2 L\left(r_{1} x_{1}+r_{2} x_{2}\right)
$$

for all $x_{1}, x_{2} \in \mathcal{X}$. Replacing $x_{1}$ and $x_{2}$ by $x / r_{1}$ and $y / r_{2}$ in (2.4), we get

$$
L(-x+y)+L(x-y)+L(x)+L(y)-L(-x)-L(-y)=2 L(x+y)
$$

for all $x, y \in \mathcal{X}$. Letting $y=-x$ in (2.5), we get that $L(-2 x)+L(2 x)=0$ for all $x \in \mathcal{X}$. So the mapping $L$ is odd. Therefore, it follows from (2.5) that the mapping $L$ is additive. Moreover, let $x \in \mathcal{X}$ and $1 \leq k \leq n$. Setting $x_{k}=x$ and $x_{l}=0$ for all $1 \leq l \leq n, l \neq k$, in (1.9) and using the oddness of $L$, we get that $L\left(r_{k} x\right)=r_{k} L(x)$.

Using the same method as in the proof of Lemma 2.1, we have an alternative result of Lemma 2.1 when $\sum_{k=1}^{n} r_{k}=0$.

Lemma 2.2. Let $x$ and $y$ be linear spaces and let $r_{1}, \ldots, r_{n}$ be real numbers with $r_{i}, r_{j} \neq 0$ for some $1 \leq i<j \leq n$. Assume that a mapping $L: \mathcal{X} \rightarrow \mathcal{y}$ with $L(0)=0$ satisfies the functional equation (1.9) for all $x_{1}, \ldots, x_{n} \in X$. Then the mapping $L$ is Cauchy additive. Moreover, $L\left(r_{k} x\right)=r_{k} L(x)$ for all $x \in \mathcal{X}$ and all $1 \leq k \leq n$.

We investigate the generalized Hyers-Ulam stability of a generalized Euler-Lagrange type additive mapping in Banach spaces.

Throughout this paper, $r_{1}, \ldots, r_{n}$ will be real numbers such that $r_{i}, r_{j} \neq 0$ for fixed $1 \leq i<j$ $\leq n$. 
Theorem 2.3. Let $f: X \rightarrow Y$ be a mapping satisfying $f(0)=0$ for which there is a function $\varphi: X^{n} \rightarrow[0, \infty)$ such that

$$
\begin{gathered}
\widetilde{\varphi_{i j}}(x, y):=\sum_{k=0}^{\infty} \frac{1}{2^{k}} \varphi(0, \ldots, \underbrace{2^{k} x}_{i t h}, 0, \ldots, \underbrace{2^{k} y}_{j t h}, 0, \ldots, 0)<\infty, \\
\lim _{k \rightarrow \infty} \frac{1}{2^{k}} \varphi\left(2^{k} x_{1}, \ldots, 2^{k} x_{n}\right)=0, \\
\left\|D_{e, r_{1}, \ldots, r_{n}} f\left(x_{1}, \ldots, x_{n}\right)\right\|_{\Upsilon} \leq \varphi\left(x_{1}, \ldots, x_{n}\right)
\end{gathered}
$$

for all $x, x_{1}, \ldots, x_{n} \in X$ and $y \in\{0, \pm x\}$. Then there exists a unique generalized Euler-Lagrange type additive mapping $L: X \rightarrow Y$ such that

$$
\begin{aligned}
\|f(x)-L(x)\|_{Y} \leq \frac{1}{4}\{ & {\left[\widetilde{\varphi_{i j}}\left(\frac{x}{r_{i}}, \frac{x}{r_{j}}\right)+2 \widetilde{\varphi_{i j}}\left(\frac{x}{2 r_{i}},-\frac{x}{2 r_{j}}\right)\right] } \\
& \left.+\left[\widetilde{\varphi_{i j}}\left(\frac{x}{r_{i}}, 0\right)+2 \widetilde{\varphi_{i j}}\left(\frac{x}{2 r_{i}}, 0\right)\right]+\left[\widetilde{\varphi_{i j}}\left(0, \frac{x}{r_{j}}\right)+2 \widetilde{\varphi_{i j}}\left(0,-\frac{x}{2 r_{j}}\right)\right]\right\}
\end{aligned}
$$

for all $x \in X$. Moreover, $L\left(r_{k} x\right)=r_{k} L(x)$ for all $x \in X$ and all $1 \leq k \leq n$.

Proof. For each $1 \leq k \leq n$ with $k \neq i, j$, let $x_{k}=0$ in (2.8), then we get the following inequality

$$
\begin{aligned}
& \left\|f\left(-r_{i} x_{i}+r_{j} x_{j}\right)+f\left(r_{i} x_{i}-r_{j} x_{j}\right)-2 f\left(r_{i} x_{i}+r_{j} x_{j}\right)+2 r_{i} f\left(x_{i}\right)+2 r_{j} f\left(x_{j}\right)\right\|_{Y} \\
& \quad \leq \varphi(0, \ldots, 0, \underbrace{x_{i}}_{i \text { th }}, 0, \ldots, 0, \underbrace{x_{j}}_{j \text { th }}, 0, \ldots, 0)
\end{aligned}
$$

for all $x_{i}, x_{j} \in X$. For convenience, set

$$
\varphi_{i j}(x, y):=\varphi(0, \ldots, 0, \underbrace{x}_{i \text { th }}, 0, \ldots, 0, \underbrace{y}_{j \text { th }}, 0, \ldots, 0)
$$

for all $x, y \in X$ and all $1 \leq i<j \leq n$. Letting $x_{i}=0$ in (2.10), we get

$$
\left\|f\left(-r_{j} x_{j}\right)-f\left(r_{j} x_{j}\right)+2 r_{j} f\left(x_{j}\right)\right\|_{Y} \leq \varphi_{i j}\left(0, x_{j}\right)
$$

for all $x_{j} \in X$. Similarly, letting $x_{j}=0$ in (2.10), we get

$$
\left\|f\left(-r_{i} x_{i}\right)-f\left(r_{i} x_{i}\right)+2 r_{i} f\left(x_{i}\right)\right\|_{Y} \leq \varphi_{i j}\left(x_{i}, 0\right)
$$


for all $x_{i} \in X$. It follows from (2.10), (2.12) and (2.13) that

$$
\begin{aligned}
& \left\|f\left(-r_{i} x_{i}+r_{j} x_{j}\right)+f\left(r_{i} x_{i}-r_{j} x_{j}\right)-2 f\left(r_{i} x_{i}+r_{j} x_{j}\right)+f\left(r_{i} x_{i}\right)+f\left(r_{j} x_{j}\right)-f\left(-r_{i} x_{i}\right)-f\left(-r_{j} x_{j}\right)\right\|_{Y} \\
& \quad \leq \varphi_{i j}\left(x_{i}, x_{j}\right)+\varphi_{i j}\left(x_{i}, 0\right)+\varphi_{i j}\left(0, x_{j}\right)
\end{aligned}
$$

for all $x_{i}, x_{j} \in X$. Replacing $x_{i}$ and $x_{j}$ by $x / r_{i}$ and $y / r_{j}$ in (2.14), we get that

$$
\begin{gathered}
\|f(-x+y)+f(x-y)-2 f(x+y)+f(x)+f(y)-f(-x)-f(-y)\|_{Y} \\
\quad \leq \varphi_{i j}\left(\frac{x}{r_{i}}, \frac{y}{r_{j}}\right)+\varphi_{i j}\left(\frac{x}{r_{i}}, 0\right)+\varphi_{i j}\left(0, \frac{y}{r_{j}}\right)
\end{gathered}
$$

for all $x, y \in X$. Putting $y=x$ in (2.15), we get

$$
\|2 f(x)-2 f(-x)-2 f(2 x)\|_{Y} \leq \varphi_{i j}\left(\frac{x}{r_{i}}, \frac{x}{r_{j}}\right)+\varphi_{i j}\left(\frac{x}{r_{i}}, 0\right)+\varphi_{i j}\left(0, \frac{x}{r_{j}}\right)
$$

for all $x \in X$. Replacing $x$ and $y$ by $x / 2$ and $-x / 2$ in (2.15), respectively, we get

$$
\|f(x)+f(-x)\|_{Y} \leq \varphi_{i j}\left(\frac{x}{2 r_{i}},-\frac{x}{2 r_{j}}\right)+\varphi_{i j}\left(\frac{x}{2 r_{i}}, 0\right)+\varphi_{i j}\left(0,-\frac{x}{2 r_{j}}\right)
$$

for all $x \in X$. It follows from (2.16) and (2.17) that

$$
\|f(2 x)-2 f(x)\|_{Y} \leq \psi(x)
$$

for all $x \in X$, where

$$
\begin{aligned}
\psi(x):=\frac{1}{2}\left\{\left[\varphi_{i j}\left(\frac{x}{r_{i}}, \frac{x}{r_{j}}\right)+2 \varphi_{i j}\left(\frac{x}{2 r_{i}},-\frac{x}{2 r_{j}}\right)\right]\right. \\
\left.+\left[\varphi_{i j}\left(\frac{x}{r_{i}}, 0\right)+2 \varphi_{i j}\left(\frac{x}{2 r_{i}}, 0\right)\right]+\left[\varphi_{i j}\left(0, \frac{x}{r_{j}}\right)+2 \varphi_{i j}\left(0,-\frac{x}{2 r_{j}}\right)\right]\right\} .
\end{aligned}
$$

It follows from (2.6) that

$$
\begin{aligned}
\sum_{k=0}^{\infty} \frac{1}{2^{k}} \psi\left(2^{k} x\right)=\frac{1}{2}\left\{\left[\widetilde{\varphi_{i j}}\left(\frac{x}{r_{i}}, \frac{x}{r_{j}}\right)+2 \widetilde{\varphi_{i j}}\left(\frac{x}{2 r_{i}},-\frac{x}{2 r_{j}}\right)\right]\right. \\
\left.+\left[\widetilde{\varphi_{i j}}\left(\frac{x}{r_{i}}, 0\right)+2 \widetilde{\varphi_{i j}}\left(\frac{x}{2 r_{i}}, 0\right)\right]+\left[\widetilde{\varphi_{i j}}\left(0, \frac{x}{r_{j}}\right)+2 \widetilde{\varphi_{i j}}\left(0,-\frac{x}{2 r_{j}}\right)\right]\right\}<\infty
\end{aligned}
$$


for all $x \in X$. Replacing $x$ by $2^{k} x$ in (2.18) and dividing both sides of (2.18) by $2^{k+1}$, we get

$$
\left\|\frac{1}{2^{k+1}} f\left(2^{k+1} x\right)-\frac{1}{2^{k}} f\left(2^{k} x\right)\right\|_{Y} \leq \frac{1}{2^{k+1}} \psi\left(2^{k} x\right)
$$

for all $x \in X$ and all $k \in \mathbb{Z}$. Therefore, we have

$$
\begin{aligned}
& \left\|\frac{1}{2^{k+1}} f\left(2^{k+1} x\right)-\frac{1}{2^{m}} f\left(2^{m} x\right)\right\|_{Y} \\
& \quad \leq \sum_{l=m}^{k}\left\|\frac{1}{2^{l+1}} f\left(2^{l+1} x\right)-\frac{1}{2^{l}} f\left(2^{l} x\right)\right\|_{Y} \leq \frac{1}{2} \sum_{l=m}^{k} \frac{1}{2^{l}} \psi\left(2^{l} x\right)
\end{aligned}
$$

for all $x \in X$ and all integers $k \geq m$. It follows from (2.20) and (2.22) that the sequence $\left\{f\left(2^{k} x\right) / 2^{k}\right\}$ is Cauchy in $Y$ for all $x \in X$, and thus converges by the completeness of $Y$. Thus we can define a mapping $L: X \rightarrow Y$ by

$$
L(x)=\lim _{k \rightarrow \infty} \frac{f\left(2^{k} x\right)}{2^{k}}
$$

for all $x \in X$. Letting $m=0$ in (2.22) and taking the limit as $k \rightarrow \infty$ in (2.22), we obtain the desired inequality (2.9).

It follows from (2.7) and (2.8) that

$$
\begin{aligned}
\left\|D_{e, r_{1}, \ldots, r_{n}} L\left(x_{1}, \ldots, x_{n}\right)\right\|_{Y} & =\lim _{k \rightarrow \infty} \frac{1}{2^{k}}\left\|D_{e, r_{1}, \ldots, r_{n}} f\left(2^{k} x_{1}, \ldots, 2^{k} x_{n}\right)\right\|_{Y} \\
& \leq \lim _{k \rightarrow \infty} \frac{1}{2^{k}} \varphi\left(2^{k} x_{1}, \ldots, 2^{k} x_{n}\right)=0
\end{aligned}
$$

for all $x_{1}, \ldots, x_{n} \in X$. Therefore, the mapping $L: X \rightarrow Y$ satisfies (1.9) and $L(0)=0$. Hence by Lemma $2.2, L$ is a generalized Euler-Lagrange type additive mapping and $L\left(r_{k} x\right)=r_{k} L(x)$ for all $x \in X$ and all $1 \leq k \leq n$.

To prove the uniqueness, let $T: X \rightarrow Y$ be another generalized Euler-Lagrange type additive mapping with $T(0)=0$ satisfying (2.9). By Lemma 2.2, the mapping $T$ is additive. Therefore, it follows from (2.9) and (2.20) that

$$
\begin{aligned}
\|L(x)-T(x)\|_{Y} & =\lim _{k \rightarrow \infty} \frac{1}{2^{k}}\left\|f\left(2^{k} x\right)-T\left(2^{k} x\right)\right\|_{Y} \leq \frac{1}{2} \lim _{k \rightarrow \infty} \frac{1}{2^{k}} \sum_{l=0}^{\infty} \frac{1}{2^{l}} \psi\left(2^{l+k} x\right) \\
& =\frac{1}{2} \lim _{k \rightarrow \infty} \sum_{l=k}^{\infty} \frac{1}{2^{l}} \psi\left(2^{l} x\right)=0 .
\end{aligned}
$$

So $L(x)=T(x)$ for all $x \in X$. 
Theorem 2.4. Let $f: X \rightarrow Y$ be a mapping satisfying $f(0)=0$ for which there is a function $\varphi: X^{n} \rightarrow[0, \infty)$ satisfying $(2.6),(2.7)$ and

$$
\left\|D_{u, r_{1}, \ldots, r_{n}} f\left(x_{1}, \ldots, x_{n}\right)\right\| \leq \varphi\left(x_{1}, \ldots, x_{n}\right)
$$

for all $x_{1}, \ldots, x_{n} \in X$ and all $u \in U(A)$. Then there exists a unique A-linear generalized EulerLagrange type additive mapping $L: X \rightarrow Y$ satisfying (2.9) for all $x \in X$. Moreover, $L\left(r_{k} x\right)=$ $r_{k} L(x)$ for all $x \in X$ and all $1 \leq k \leq n$.

Proof. By Theorem 2.3, there exists a unique generalized Euler-Lagrange type additive mapping $L: X \rightarrow Y$ satisfying (2.9) and moreover $L\left(r_{k} x\right)=r_{k} L(x)$ for all $x \in X$ and all $1 \leq k \leq n$.

By the assumption, for each $u \in U(A)$, we get

$$
\begin{aligned}
\left\|D_{u, r_{1}, \ldots, r_{n}} L(0, \ldots, 0, \underbrace{x}_{i \text { th }}, 0 \cdots, 0)\right\|_{Y} & =\lim _{k \rightarrow \infty} \frac{1}{2^{k}}\left\|D_{u, r_{1}, \ldots, r_{n}} f(0, \ldots, 0, \underbrace{2^{k} x}_{i \text { th }}, 0 \cdots, 0)\right\|_{Y} \\
& \leq \lim _{k \rightarrow \infty} \frac{1}{2^{k}} \varphi(0, \ldots, 0, \underbrace{2^{k} x}_{i \text { th }}, 0 \cdots, 0)=0
\end{aligned}
$$

for all $x \in X$. So

$$
r_{i} u L(x)=L\left(r_{i} u x\right)
$$

for all $u \in U(A)$ and all $x \in X$. Since $L\left(r_{i} x\right)=r_{i} L(x)$ for all $x \in X$ and $r_{i} \neq 0$,

$$
L(u x)=u L(x)
$$

for all $u \in U(A)$ and all $x \in X$.

By the same reasoning as in the proofs of $[41,43]$,

$$
L(a x+b y)=L(a x)+L(b y)=a L(x)+b L(y)
$$

for all $a, b \in A(a, b \neq 0)$ and all $x, y \in X$. Since $L(0 x)=0=0 L(x)$ for all $x \in X$, the unique generalized Euler-Lagrange type additive mapping $L: X \rightarrow Y$ is an $A$-linear mapping.

Corollary 2.5. Let $\delta \geq 0,\left\{\epsilon_{k}\right\}_{k \in J}$ and $\left\{p_{k}\right\}_{k \in J}$ be real numbers such that $\epsilon_{k} \geq 0$ and $0<p_{k}<1$ for all $k \in J$, where $J \subseteq\{1,2, \ldots, n\}$. Assume that a mapping $f: X \rightarrow Y$ with $f(0)=0$ satisfies the inequality

$$
\left\|D_{u, r_{1}, \ldots, r_{n}} f\left(x_{1}, \ldots, x_{n}\right)\right\|_{Y} \leq \delta+\sum_{k \in J} \epsilon_{k}\left\|x_{k}\right\|_{X}^{p_{k}}
$$


for all $x_{1}, \ldots, x_{n} \in X$ and all $u \in U(A)$. Then there exists a unique A-linear generalized EulerLagrange type additive mapping $L: X \rightarrow Y$ such that

$$
\|f(x)-L(x)\|_{Y} \leq \begin{cases}M_{i j}(x), & i, j \in J ; \\ M_{i}(x), & i \in J, j \notin J ; \\ M_{j}(x), & j \in J, i \notin J \\ M, & i, j \notin J .\end{cases}
$$

for all $x \in X$, where

$$
\begin{gathered}
M_{i j}(x)=\frac{9}{2} \delta+\sum_{k \in\{i, j\}} \frac{\left(1+2^{1-p_{k}}\right) \epsilon_{k}}{\left(2-2^{p_{k}}\right) r_{k}^{p_{k}}}\|x\|_{X}^{p_{k}} \\
M_{i}(x)=\frac{9}{2} \delta+\frac{\left(1+2^{1-p_{i}}\right) \epsilon_{i}}{\left(2-2^{p_{i}}\right) r_{i}^{p_{i}}}\|x\|_{X^{\prime}}^{p_{i}} \\
M_{j}(x)=\frac{9}{2} \delta+\frac{\left(1+2^{1-p_{j}}\right) \epsilon_{j}}{\left(2-2^{p_{j}}\right) r_{j}^{p_{j}}}\|x\|_{X^{\prime}}^{p_{j}} \quad M=\frac{9}{2} \delta .
\end{gathered}
$$

Moreover, $L\left(r_{k} x\right)=r_{k} L(x)$ for all $x \in X$ and all $1 \leq k \leq n$.

Proof. Define $\varphi\left(x_{1}, \ldots, x_{n}\right):=\delta+\sum_{k \in J} \epsilon_{k}\left\|x_{k}\right\|_{X}^{p_{k}}$, and apply Theorem 2.4.

Corollary 2.6. Let $\delta, \epsilon \geq 0, p, q>0$ with $\lambda=p+q<1$. Assume that a mapping $f: X \rightarrow Y$ with $f(0)=0$ satisfies the inequality

$$
\left\|D_{u, r_{1}, \ldots, r_{n}} f\left(x_{1}, \ldots, x_{n}\right)\right\|_{Y} \leq \delta+\epsilon\left\|x_{i}\right\|_{X}^{p}\left\|x_{j}\right\|_{X}^{q}
$$

for all $x_{1}, \ldots, x_{n} \in X$ and all $u \in U(A)$. Then there exists a unique A-linear generalized EulerLagrange type additive mapping $L: X \rightarrow Y$ such that

$$
\|f(x)-L(x)\|_{Y} \leq \frac{9}{2} \delta+\frac{\left(1+2^{1-\lambda}\right) \epsilon}{2\left(2-2^{\lambda}\right) r_{i}^{p} r_{j}^{q}}\|x\|_{X}^{\lambda}
$$

for all $x \in X$. Moreover, $L\left(r_{k} x\right)=r_{k} L(x)$ for all $x \in X$ and all $1 \leq k \leq n$.

Proof. Define $\varphi\left(x_{1}, \ldots, x_{n}\right):=\delta+\epsilon\left\|x_{i}\right\|_{X}^{p}\left\|x_{j}\right\|_{X}^{q}$. Applying Theorem 2.4, we obtain the desired result. 
Theorem 2.7. Let $f: X \rightarrow Y$ be a mapping satisfying $f(0)=0$ for which there is a function $\phi: X^{n} \rightarrow[0, \infty)$ such that

$$
\begin{gathered}
\widetilde{\phi_{i j}}(x, y):=\sum_{k=1}^{\infty} 2^{k} \phi(0, \ldots, \underbrace{\frac{x}{2^{k}}}_{i t h}, 0, \ldots, \underbrace{\frac{y}{2^{k}}}_{j \text { th }}, 0, \ldots, 0)<\infty, \\
\lim _{k \rightarrow \infty} 2^{k} \phi\left(\frac{x_{1}}{2^{k}}, \ldots, \frac{x_{n}}{2^{k}}\right)=0, \\
\left\|D_{e, r_{1}, \ldots, r_{n}} f\left(x_{1}, \ldots, x_{n}\right)\right\|_{Y} \leq \phi\left(x_{1}, \ldots, x_{n}\right)
\end{gathered}
$$

for all $x, x_{1}, \ldots, x_{n} \in X$ and $y \in\{0, \pm x\}$. Then there exists a unique generalized Euler-Lagrange type additive mapping $L: X \rightarrow Y$ such that

$$
\begin{aligned}
\|f(x)-L(x)\|_{Y} \leq \frac{1}{4}\{ & {\left[\widetilde{\phi_{i j}}\left(\frac{x}{r_{i}}, \frac{x}{r_{j}}\right)+2 \widetilde{\phi_{i j}}\left(\frac{x}{2 r_{i}},-\frac{x}{2 r_{j}}\right)\right] } \\
& \left.+\left[\widetilde{\phi_{i j}}\left(\frac{x}{r_{i}}, 0\right)+2 \widetilde{\phi_{i j}}\left(\frac{x}{2 r_{i}}, 0\right)\right]+\left[\widetilde{\phi_{i j}}\left(0, \frac{x}{r_{j}}\right)+2 \widetilde{\phi_{i j}}\left(0,-\frac{x}{2 r_{j}}\right)\right]\right\}
\end{aligned}
$$

for all $x \in X$. Moreover, $L\left(r_{k} x\right)=r_{k} L(x)$ for all $x \in X$ and all $1 \leq k \leq n$.

Proof. By a similar method to the proof of Theorem 2.3, we have the following inequality

$$
\|f(2 x)-2 f(x)\|_{Y} \leq \Psi(x)
$$

for all $x \in X$, where

$$
\begin{aligned}
\Psi(x):=\frac{1}{2}\{ & {\left[\phi_{i j}\left(\frac{x}{r_{i}}, \frac{x}{r_{j}}\right)+2 \phi_{i j}\left(\frac{x}{2 r_{i}},-\frac{x}{2 r_{j}}\right)\right] } \\
& \left.+\left[\phi_{i j}\left(\frac{x}{r_{i}}, 0\right)+2 \phi_{i j}\left(\frac{x}{2 r_{i}}, 0\right)\right]+\left[\phi_{i j}\left(0, \frac{x}{r_{j}}\right)+2 \phi_{i j}\left(0,-\frac{x}{2 r_{j}}\right)\right]\right\} .
\end{aligned}
$$

It follows from (2.36) that

$$
\begin{aligned}
\sum_{k=1}^{\infty} 2^{k} \Psi\left(\frac{x}{2^{k}}\right)=\frac{1}{2}\{ & {\left[\widetilde{\phi_{i j}}\left(\frac{x}{r_{i}}, \frac{x}{r_{j}}\right)+2 \widetilde{\phi_{i j}}\left(\frac{x}{2 r_{i}},-\frac{x}{2 r_{j}}\right)\right] } \\
& \left.+\left[\widetilde{\phi_{i j}}\left(\frac{x}{r_{i}}, 0\right)+2 \widetilde{\phi_{i j}}\left(\frac{x}{2 r_{i}}, 0\right)\right]+\left[\widetilde{\phi_{i j}}\left(0, \frac{x}{r_{j}}\right)+2 \widetilde{\phi_{i j}}\left(0,-\frac{x}{2 r_{j}}\right)\right]\right\}<\infty
\end{aligned}
$$


for all $x \in X$. Replacing $x$ by $x / 2^{k+1}$ in (2.40) and multiplying both sides of (2.40) by $2^{k}$, we get

$$
\left\|2^{k+1} f\left(\frac{x}{2^{k+1}}\right)-2^{k} f\left(\frac{x}{2^{k}}\right)\right\|_{Y} \leq 2^{k} \Psi\left(\frac{x}{2^{k+1}}\right)
$$

for all $x \in X$ and all $k \in \mathbb{Z}$. Therefore, we have

$$
\begin{array}{rl}
\| 2^{k+1} & f\left(\frac{x}{2^{k+1}}\right)-2^{m} f\left(\frac{x}{2^{m}}\right) \|_{Y} \\
& \leq \sum_{l=m}^{k}\left\|2^{l+1} f\left(\frac{x}{2^{l+1}}\right)-2^{l} f\left(\frac{x}{2^{l}}\right)\right\|_{Y} \leq \sum_{l=m}^{k} 2^{l} \Psi\left(\frac{x}{2^{l+1}}\right)
\end{array}
$$

for all $x \in X$ and all integers $k \geq m$. It follows from (2.42) and (2.44) that the sequence $\left\{2^{k} f\left(x / 2^{k}\right)\right\}$ is Cauchy in $Y$ for all $x \in X$, and thus converges by the completeness of $Y$. Thus we can define a mapping $L: X \rightarrow Y$ by

$$
L(x)=\lim _{k \rightarrow \infty} 2^{k} f\left(\frac{x}{2^{k}}\right)
$$

for all $x \in X$. Letting $m=0$ in (2.44) and taking the limit as $k \rightarrow \infty$ in (2.44), we obtain the desired inequality (2.39).

The rest of the proof is similar to the proof of Theorem 2.3.

Theorem 2.8. Let $f: X \rightarrow Y$ be a mapping with $f(0)=0$ for which there is a function $\phi: X^{n} \rightarrow$ $[0, \infty)$ satisfying $(2.36),(2.37)$ and

$$
\left\|D_{u, r_{1}, \ldots, r_{n}} f\left(x_{1}, \ldots, x_{n}\right)\right\| \leq \phi\left(x_{1}, \ldots, x_{n}\right)
$$

for all $x_{1}, \ldots, x_{n} \in X$ and all $u \in U(A)$. Then there exists a unique A-linear generalized EulerLagrange type additive mapping $L: X \rightarrow Y$ satisfying (2.39) for all $x \in X$. Moreover, $L\left(r_{k} x\right)=$ $r_{k} L(x)$ for all $x \in X$ and all $1 \leq k \leq n$.

Proof. The proof is similar to the proof of Theorem 2.4.

Corollary 2.9. Let $\left\{\epsilon_{k}\right\}_{k \in J}$ and $\left\{p_{k}\right\}_{k \in J}$ be real numbers such that $\epsilon_{k} \geq 0$ and $p_{k}>1$ for all $k \in J$, where $J \subseteq\{1,2, \ldots, n\}$. Assume that a mapping $f: X \rightarrow Y$ with $f(0)=0$ satisfies the inequality

$$
\left\|D_{u, r_{1}, \ldots, r_{n}} f\left(x_{1}, \ldots, x_{n}\right)\right\|_{Y} \leq \sum_{k \in J} \epsilon_{k}\left\|x_{k}\right\|_{X}^{p_{k}}
$$


for all $x_{1}, \ldots, x_{n} \in X$ and all $u \in U(A)$. Then there exists a unique A-linear generalized EulerLagrange type additive mapping $L: X \rightarrow Y$ such that

$$
\|f(x)-L(x)\|_{Y} \leq \begin{cases}N_{i j}(x), & i, j \in J ; \\ N_{i}(x), & i \in J, j \notin J \\ N_{j}(x), & j \in J, i \notin J \\ N, & i, j \notin J .\end{cases}
$$

for all $x \in X$, where

$$
\begin{gathered}
N_{i j}(x)=\sum_{k \in\{i, j\}} \frac{\left(1+2^{1-p_{k}}\right) \epsilon_{k}}{\left(2^{p_{k}}-2\right) r_{k}^{p_{k}}}\|x\|_{X^{\prime}}^{p_{k}} \\
N_{i}(x)=\frac{\left(1+2^{1-p_{i}}\right) \epsilon_{i}}{\left(2^{p_{i}}-2\right) r_{i}^{p_{i}}}\|x\|_{X^{\prime}}^{p_{i}} \\
N_{j}(x)=\frac{\left(1+2^{1-p_{j}}\right) \epsilon_{j}}{\left(2^{p_{j}}-2\right) r_{j}^{p_{j}}}\|x\|_{X}^{p_{j}}
\end{gathered}
$$

Moreover, $L\left(r_{k} x\right)=r_{k} L(x)$ for all $x \in X$ and all $1 \leq k \leq n$.

Proof. Define $\phi\left(x_{1}, \ldots, x_{n}\right):=\sum_{k \in J} \epsilon_{k}\left\|x_{k}\right\|_{X}^{p_{k}}$. Applying Theorem 2.8, we obtain the desired result.

Corollary 2.10. Let $\epsilon \geq 0, p, q>0$ with $\lambda=p+q>1$. Assume that a mapping $f: X \rightarrow Y$ with $f(0)=0$ satisfies the inequality

$$
\left\|D_{u, r_{1}, \ldots, r_{n}} f\left(x_{1}, \ldots, x_{n}\right)\right\|_{Y} \leq \epsilon\left\|x_{i}\right\|_{X}^{p}\left\|x_{j}\right\|_{X}^{q}
$$

for all $x_{1}, \ldots, x_{n} \in X$ and all $u \in U(A)$. Then there exists a unique A-linear generalized EulerLagrange type additive mapping $L: X \rightarrow Y$ such that

$$
\|f(x)-L(x)\|_{Y} \leq \frac{\left(1+2^{1-\lambda}\right) \epsilon}{2\left(2^{\lambda}-2\right) r_{i}^{p} r_{j}^{q}}\|x\|_{X}^{\lambda}
$$

for all $x \in X$. Moreover, $L\left(r_{k} x\right)=r_{k} L(x)$ for all $x \in X$ and all $1 \leq k \leq n$.

Proof. Define $\phi\left(x_{1}, \ldots, x_{n}\right):=\epsilon\left\|x_{i}\right\|_{X}^{p}\left\|x_{j}\right\|_{X}^{q}$. Applying Theorem 2.8, we obtain the desired result.

Remark 2.11. In Theorems 2.7 and 2.8 and Corollaries 2.9 and 2.10 one can assume that $\sum_{k=1}^{n} r_{k} \neq 0$ instead of $f(0)=0$.

For the case $p_{1}=\cdots=p_{n}=1$ in Corollaries 2.5 and 2.9, using an idea from the example of Gajda [56], we have the following counterexample. 
Example 2.12. Let $\phi: \mathbb{C} \rightarrow \mathbb{C}$ be defined by

$$
\phi(x):= \begin{cases}x & \text { for }|x|<1 \\ 1 & \text { otherwise }\end{cases}
$$

Consider the function $f: \mathbb{C} \rightarrow \mathbb{C}$ by the formula

$$
f(x):=\sum_{n=0}^{\infty} 2^{-n} \phi\left(2^{n} x\right)
$$

It is clear that $f$ is continuous and bounded by 2 on $\mathbb{C}$. We prove that

$$
\left|D_{\mu, r_{1}, \ldots, r_{n}} f\left(x_{1}, \ldots, x_{n}\right)\right| \leq 8\left(n+\sum_{i=1}^{n}\left|r_{i}\right|\right) \sum_{i=1}^{n}\left(\left|r_{i}\right|+1\right)\left|x_{i}\right|
$$

for all $x_{1}, \ldots, x_{n} \in \mathbb{C}$ and all $\mu \in U(\mathbb{C})=\{\lambda \in \mathbb{C}:|\lambda|=1\}$. If $\sum_{i=1}^{n}\left(\left|r_{i}\right|+1\right)\left|x_{i}\right|=0$ or $\sum_{i=1}^{n}\left(\left|r_{i}\right|+1\right)\left|x_{i}\right| \geq 1$, then

$$
\left|D_{\mu, r_{1}, \ldots, r_{n}} f\left(x_{1}, \ldots, x_{n}\right)\right| \leq 4 n+4 \sum_{i=1}^{n}\left|r_{i}\right| \leq 4\left(n+\sum_{i=1}^{n}\left|r_{i}\right|\right) \sum_{i=1}^{n}\left(\left|r_{i}\right|+1\right)\left|x_{i}\right| .
$$

Now suppose that $0<\sum_{i=1}^{n}\left(\left|r_{i}\right|+1\right)\left|x_{i}\right|<1$. Then there exists a nonnegative integer $k$ such that

$$
\frac{1}{2^{k+1}} \leq \sum_{i=1}^{n}\left(\left|r_{i}\right|+1\right)\left|x_{i}\right|<\frac{1}{2^{k}} .
$$

Therefore

$$
2^{k}\left|-\mu r_{j} x_{j}+\sum_{1 \leq i \leq n, i \neq j} \mu r_{i} x_{i}\right|, 2^{k}\left|\sum_{i=1}^{n} \mu r_{i} x_{i}\right|, 2^{k}\left|x_{1}\right|, \ldots, 2^{k}\left|x_{n}\right| \in(-1,1) .
$$

Hence

$$
2^{m}\left|-\mu r_{j} x_{j}+\sum_{1 \leq i \leq n, i \neq j} \mu r_{i} x_{i}\right|, 2^{m}\left|\sum_{i=1}^{n} \mu r_{i} x_{i}\right|, 2^{m}\left|x_{1}\right|, \ldots, 2^{m}\left|x_{n}\right| \in(-1,1)
$$


for all $m=0,1, \ldots, k$. From the definition of $f$ and (2.56), we have

$$
\begin{aligned}
\left|D_{\mu, r_{1}, \ldots, r_{n}} f\left(x_{1}, \ldots, x_{n}\right)\right| & \leq 4\left(n+\sum_{i=1}^{n}\left|r_{i}\right|\right) \sum_{m=k+1}^{\infty} \frac{1}{2^{m}} \\
& =8\left(n+\sum_{i=1}^{n}\left|r_{i}\right|\right) \frac{1}{2^{k+1}} \\
& \leq 8\left(n+\sum_{i=1}^{n}\left|r_{i}\right|\right) \sum_{i=1}^{n}\left(\left|r_{i}\right|+1\right)\left|x_{i}\right| .
\end{aligned}
$$

Therefore $f$ satisfies (2.54). Let $L: \mathbb{C} \rightarrow \mathbb{C}$ be an additive mapping such that

$$
|f(x)-L(x)| \leq \beta|x|
$$

for all $x \in \mathbb{C}$. Then there exists a constant $c \in \mathbb{C}$ such that $L(x)=c x$ for all rational numbers $x$. So we have

$$
|f(x)| \leq(\beta+|c|)|x|
$$

for all rational numbers $x$. Let $m \in \mathbb{N}$ with $m>\beta+|c|$. If $x$ is a rational number in $\left(0,2^{1-m}\right)$, then $2^{n} x \in(0,1)$ for all $n=0,1, \ldots, m-1$. So

$$
f(x) \geq \sum_{n=0}^{m-1} 2^{-n} \phi\left(2^{n} x\right)=m x>(\beta+|c|)|x|
$$

which contradicts with (2.61).

\section{Homomorphisms in Unital $C^{*}$-Algebras}

In this section, we investigate $C^{*}$-algebra homomorphisms in unital $C^{*}$-algebras. We will use the following lemma in the proof of the next theorem.

Lemma 3.1 (see [43]). Let $f: A \rightarrow B$ be an additive mapping such that $f(\mu x)=\mu f(x)$ for all $x \in A$ and all $\mu \in \mathbb{S}^{1}:=\{\lambda \in \mathbb{C}:|\lambda|=1\}$. Then the mapping $f: A \rightarrow B$ is $\mathbb{C}$-linear. 
Theorem 3.2. Let $\epsilon \geq 0$ and $\left\{p_{k}\right\}_{k \in J}$ be real numbers such that $p_{k}>0$ for all $k \in J$, where $J \subseteq$ $\{1,2, \ldots, n\}$ and $|J| \geq 3$. Let $f: A \rightarrow B$ be a mapping with $f(0)=0$ for which there is a function $\varphi: A^{n} \rightarrow[0, \infty)$ satisfying $(2.7)$ and

$$
\begin{gathered}
\left\|D_{\mu, r_{1}, \ldots, r_{n}} f\left(x_{1}, \ldots, x_{n}\right)\right\|_{B} \leq \epsilon \prod_{k \in J}\left\|x_{k}\right\|_{A}^{p_{k}}, \\
\left\|f\left(2^{k} u^{*}\right)-f\left(2^{k} u\right)^{*}\right\|_{B} \leq \varphi(\underbrace{2^{k} u, \ldots, 2^{k} u}_{n \text { times }}), \\
\left\|f\left(2^{k} u x\right)-f\left(2^{k} u\right) f(x)\right\|_{B} \leq \varphi(\underbrace{2^{k} u x, \ldots, 2^{k} u x}_{n \text { times }})
\end{gathered}
$$

for all $x, x_{1}, \ldots, x_{n} \in A$, for all $u \in U(A)$, all $k \in \mathbb{N}$ and all $\mu \in \mathbb{S}^{1}$. Then the mapping $f: A \rightarrow B$ is a $C^{*}$-algebra homomorphism.

Proof. Since $|J| \geq 3$, letting $\mu=1$ and $x_{k}=0$ for all $1 \leq k \leq n, k \neq i, j$, in (3.1), we get

$$
f\left(-r_{i} x_{i}+r_{j} x_{j}\right)+f\left(r_{i} x_{i}-r_{j} x_{j}\right)+2 r_{i} f\left(x_{i}\right)+2 r_{j} f\left(x_{j}\right)=2 f\left(r_{i} x_{i}+r_{j} x_{j}\right)
$$

for all $x_{i}, x_{j} \in A$. By the same reasoning as in the proof of Lemma 2.1, the mapping $f$ is additive and $f\left(r_{k} x\right)=r_{k} f(x)$ for all $x \in A$ and $k=i, j$. So by letting $x_{i}=x$ and $x_{k}=0$ for all $1 \leq k \leq n, k \neq i$, in (3.1), we get that $f(\mu x)=\mu f(x)$ for all $x \in A$ and all $\mu \in \mathbb{S}^{1}$. Therefore, by Lemma 3.1, the mapping $f$ is $\mathbb{C}$-linear. Hence it follows from (2.7), (3.2) and (3.3) that

$$
\begin{aligned}
\left\|f\left(u^{*}\right)-f(u)^{*}\right\|_{B} & =\lim _{k \rightarrow \infty} \frac{1}{2^{k}}\left\|f\left(2^{k} u^{*}\right)-f\left(2^{k} u\right)^{*}\right\|_{B} \\
& \leq \lim _{k \rightarrow \infty} \frac{1}{2^{k}} \varphi(\underbrace{2^{k} u, \ldots, 2^{k} u}_{n \text { times }})=0, \\
\|f(u x)-f(u) f(x)\|_{B} & =\lim _{k \rightarrow \infty} \frac{1}{2^{k}}\left\|f\left(2^{k} u x\right)-f\left(2^{k} u\right) f(x)\right\|_{B} \\
& \leq \lim _{k \rightarrow \infty} \frac{1}{2^{k}} \varphi(\underbrace{2^{k} u x, \ldots, 2^{k} u x}_{n \text { times }})=0
\end{aligned}
$$

for all $x \in A$ and all $u \in U(A)$. So $f\left(u^{*}\right)=f(u)^{*}$ and $f(u x)=f(u) f(x)$ for all $x \in A$ and all $u \in U(A)$. Since $f$ is $\mathbb{C}$-linear and each $x \in A$ is a finite linear combination of unitary elements 
(see [57]), that is, $x=\sum_{k=1}^{m} \lambda_{k} u_{k}$, where $\lambda_{k} \in \mathbb{C}$ and $u_{k} \in U(A)$ for all $1 \leq k \leq n$, we have

$$
\begin{aligned}
f\left(x^{*}\right) & =f\left(\sum_{k=1}^{m} \overline{\lambda_{k}} u_{k}^{*}\right)=\sum_{k=1}^{m} \overline{\lambda_{k}} f\left(u_{k}^{*}\right)=\sum_{k=1}^{m} \overline{\lambda_{k}} f\left(u_{k}\right)^{*} \\
& =\left(\sum_{k=1}^{m} \lambda_{k} f\left(u_{k}\right)\right)^{*}=f\left(\sum_{k=1}^{m} \lambda_{k} u_{k}\right)^{*}=f(x)^{*}, \\
f(x y) & =f\left(\sum_{k=1}^{m} \lambda_{k} u_{k} y\right)=\sum_{k=1}^{m} \lambda_{k} f\left(u_{k} y\right) \\
& =\sum_{k=1}^{m} \lambda_{k} f\left(u_{k}\right) f(y)=f\left(\sum_{k=1}^{m} \lambda_{k} u_{k}\right) f(y)=f(x) f(y)
\end{aligned}
$$

for all $x, y \in A$. Therefore, the mapping $f: A \rightarrow B$ is a $C^{*}$-algebra homomorphism, as desired.

The following theorem is an alternative result of Theorem 3.2.

Theorem 3.3. Let $\epsilon \geq 0$ and $\left\{p_{k}\right\}_{k \in J}$ be real numbers such that $p_{k}>0$ for all $k \in J$, where $J \subseteq$ $\{1,2, \ldots, n\}$ and $|J| \geq 3$. Let $f: A \rightarrow B$ be a mapping with $f(0)=0$ for which there is a function $\varphi: A^{n} \rightarrow[0, \infty)$ satisfying $(2.37)$ and

$$
\begin{gathered}
\left\|D_{\mu, r_{1}, \ldots, r_{n}} f\left(x_{1}, \ldots, x_{n}\right)\right\|_{B} \leq \epsilon \prod_{k \in J}\left\|x_{k}\right\|_{A}^{p_{k}} \\
\left\|f\left(\frac{u^{*}}{2^{k}}\right)-f\left(\frac{u}{2^{k}}\right)^{*}\right\|_{B} \leq \phi(\underbrace{\frac{u}{2^{k}}, \ldots, \frac{u}{2^{k}}}_{n \text { times }}), \\
\left\|f\left(\frac{u x}{2^{k}}\right)-f\left(\frac{u}{2^{k}}\right) f(x)\right\|_{B} \leq \phi(\underbrace{\frac{u x}{2^{k}}, \ldots, \frac{u x}{2^{k}}}_{n \text { times }})
\end{gathered}
$$

for all $x, x_{1}, \ldots, x_{n} \in A$, for all $u \in U(A)$, all $k \in \mathbb{N}$ and all $\mu \in \mathbb{S}^{1}$. Then the mapping $f: A \rightarrow B$ is a $C^{*}$-algebra homomorphism.

Remark 3.4. In Theorems 3.2 and 3.3, one can assume that $\sum_{k=1}^{n} r_{k} \neq 0$ instead of $f(0)=0$.

Theorem 3.5. Let $f: A \rightarrow B$ be a mapping with $f(0)=0$ for which there is a function $\varphi: A^{n} \rightarrow$ $[0, \infty)$ satisfying $(2.6),(2.7),(3.2),(3.3)$ and

$$
\left\|D_{\mu, r_{1}, \ldots, r_{n}} f\left(x_{1}, \ldots, x_{n}\right)\right\|_{B} \leq \varphi\left(x_{1}, \ldots, x_{n}\right),
$$

for all $x_{1}, \ldots, x_{n} \in A$ and all $\mu \in \mathbb{S}^{1}$. Assume that $\lim _{k \rightarrow \infty}\left(1 / 2^{k}\right) f\left(2^{k} e\right)$ is invertible. Then the mapping $f: A \rightarrow B$ is a $C^{*}$-algebra homomorphism. 
Proof. Consider the $C^{*}$-algebras $A$ and $B$ as left Banach modules over the unital $C^{*}$-algebra $\mathbb{C}$. By Theorem 2.4, there exists a unique $\mathbb{C}$-linear generalized Euler-Lagrange type additive mapping $H: A \rightarrow B$ defined by

$$
H(x)=\lim _{k \rightarrow \infty} \frac{1}{2^{k}} f\left(2^{k} x\right)
$$

for all $x \in A$. Therefore, by (2.7), (3.2) and (3.3), we get

$$
\begin{aligned}
\left\|H\left(u^{*}\right)-H(u)^{*}\right\|_{B} & =\lim _{k \rightarrow \infty} \frac{1}{2^{k}}\left\|f\left(2^{k} u^{*}\right)-f\left(2^{k} u\right)^{*}\right\|_{B} \\
& \leq \lim _{k \rightarrow \infty} \frac{1}{2^{k}} \varphi(\underbrace{2^{k} u, \ldots, 2^{k} u}_{n \text { times }})=0, \\
\|H(u x)-H(u) f(x)\|_{B} & =\lim _{k \rightarrow \infty} \frac{1}{2^{k}}\left\|f\left(2^{k} u x\right)-f\left(2^{k} u\right) f(x)\right\|_{B} \\
& \leq \lim _{k \rightarrow \infty} \frac{1}{2^{k}} \varphi(\underbrace{2^{k} u x, \ldots, 2^{k} u x}_{n \text { times }})=0
\end{aligned}
$$

for all $u \in U(A)$ and for all $x \in A$. So $H\left(u^{*}\right)=H(u)^{*}$ and $H(u x)=H(u) f(x)$ for all $u \in U(A)$ and all $x \in A$. Therefore, by the additivity of $H$ we have

$$
H(u x)=\lim _{k \rightarrow \infty} \frac{1}{2^{k}} H\left(2^{k} u x\right)=H(u) \lim _{k \rightarrow \infty} \frac{1}{2^{k}} f\left(2^{k} x\right)=H(u) H(x)
$$

for all $u \in U(A)$ and all $x \in A$. Since $H$ is $\mathbb{C}$-linear and each $x \in A$ is a finite linear combination of unitary elements, that is, $x=\sum_{k=1}^{m} \lambda_{k} u_{k}$, where $\lambda_{k} \in \mathbb{C}$ and $u_{k} \in U(A)$ for all $1 \leq k \leq n$, it follows from (3.11) that

$$
\begin{aligned}
H(x y) & =H\left(\sum_{k=1}^{m} \lambda_{k} u_{k} y\right)=\sum_{k=1}^{m} \lambda_{k} H\left(u_{k} y\right) \\
& =\sum_{k=1}^{m} \lambda_{k} H\left(u_{k}\right) H(y)=H\left(\sum_{k=1}^{m} \lambda_{k} u_{k}\right) H(y)=H(x) H(y) \\
H\left(x^{*}\right) & =H\left(\sum_{k=1}^{m} \overline{\lambda_{k}} u_{k}^{*}\right)=\sum_{k=1}^{m} \overline{\lambda_{k}} H\left(u_{k}^{*}\right)=\sum_{k=1}^{m} \overline{\lambda_{k}} H\left(u_{k}\right)^{*} \\
& =\left(\sum_{k=1}^{m} \lambda_{k} H\left(u_{k}\right)\right)^{*}=H\left(\sum_{k=1}^{m} \lambda_{k} u_{k}\right)^{*}=H(x)^{*}
\end{aligned}
$$

for all $x, y \in A$. Since $H(e)=\lim _{k \rightarrow \infty}\left(1 / 2^{k}\right) f\left(2^{k} e\right)$ is invertible and

$$
H(e) H(y)=H(e y)=H(e) f(y)
$$


for all $y \in A, H(y)=f(y)$ for all $y \in A$, therefore, the mapping $f: A \rightarrow B$ is a $C^{*}$-algebra homomorphism.

The following theorem is an alternative result of Theorem 3.5.

Theorem 3.6. Let $f: A \rightarrow B$ be a mapping with $f(0)=0$ for which there is a function $\phi: A^{n} \rightarrow$ $[0, \infty)$ satisfying $(2.36),(2.37),(3.7)$ and

$$
\left\|D_{\mu, r_{1}, \ldots, r_{n}} f\left(x_{1}, \ldots, x_{n}\right)\right\|_{B} \leq \phi\left(x_{1}, \ldots, x_{n}\right),
$$

for all $x_{1}, \ldots, x_{n} \in A$ and all $\mu \in \mathbb{S}^{1}$. Assume that $\lim _{k \rightarrow \infty} 2^{k} f\left(e / 2^{k}\right)$ is invertible. Then the mapping $f: A \rightarrow B$ is a $C^{*}$-algebra homomorphism.

Corollary 3.7. Let $\left\{\epsilon_{k}\right\}_{k \in J}$ and $\left\{p_{k}\right\}_{k \in J}$ be real numbers such that $\epsilon_{k} \geq 0$ and $p_{k}>1\left(0<p_{k}<1\right)$ for all $k \in J$, where $J \subseteq\{1,2, \ldots, n\}$. Assume that a mapping $f: A \rightarrow B$ with $f(0)=0$ satisfies the inequalities

$$
\begin{gathered}
\left\|D_{\mu, r_{1}, \ldots, r_{n}} f\left(x_{1}, \ldots, x_{n}\right)\right\|_{B} \leq \sum_{k \in J} \epsilon_{k}\left\|x_{k}\right\|_{A}^{p_{k}}, \\
\left\|f\left(\frac{u^{*}}{2^{m}}\right)-f\left(\frac{u}{2^{m}}\right)^{*}\right\|_{B} \leq \sum_{k \in J} \frac{\epsilon_{k}}{2^{m p p_{k}}} \\
\left(\text { resp., }\left\|f\left(2^{m} u^{*}\right)-f\left(2^{m} u\right)^{*}\right\|_{B} \leq \sum_{k \in J} \epsilon_{k} 2^{m p_{k}}\right), \\
\left\|f\left(\frac{u x}{2^{m}}\right)-f\left(\frac{u}{2^{m}}\right) f(x)\right\|_{B} \leq \sum_{k \in J} \frac{\epsilon_{k}}{2^{m p_{k}}}\|x\|_{A}^{p_{k}} \\
\left(r e s p .,\left\|f\left(2^{m} u x\right)-f\left(2^{m} u\right) f(x)\right\|_{B} \leq \sum_{k \in J} \epsilon_{k} 2^{m p_{k}}\|x\|_{A}^{p_{k}}\right),
\end{gathered}
$$

for all $x_{1}, \ldots, x_{n} \in A$, all $u \in U(A)$, all $m \in \mathbb{N}$ and all $\mu \in \mathbb{S}^{1}$. Assume that $\lim _{k \rightarrow \infty} 2^{k} f\left(e / 2^{k}\right)\left(\right.$ resp., $\left.\lim _{k \rightarrow \infty}\left(1 / 2^{k}\right) f\left(2^{k} e\right)\right)$ is invertible. Then the mapping $f: A \rightarrow B$ is a $C^{*}$-algebra homomorphism.

Proof. The result follows from Theorem 3.6 (resp., Theorem 3.5).

Remark 3.8. In Theorem 3.6 and Corollary 3.7, one can assume that $\sum_{k=1}^{n} r_{k} \neq 0$ instead of $f(0)=0$.

Theorem 3.9. Let $f: A \rightarrow B$ be a mapping with $f(0)=0$ for which there is a function $\varphi: A^{n} \rightarrow$ $[0, \infty)$ satisfying $(2.6),(2.7),(3.2),(3.3)$ and

$$
\left\|D_{\mu, r_{1}, \ldots, r_{n}} f\left(x_{1}, \ldots, x_{n}\right)\right\|_{B} \leq \varphi\left(x_{1}, \ldots, x_{n}\right),
$$


for $\mu=i, 1$ and all $x_{1}, \ldots, x_{n} \in A$. Assume that $\lim _{k \rightarrow \infty}\left(1 / 2^{k}\right) f\left(2^{k} e\right)$ is invertible and for each fixed $x \in A$ the mapping $t \mapsto f(t x)$ is continuous in $t \in \mathbb{R}$. Then the mapping $f: A \rightarrow B$ is a $C^{*}$-algebra homomorphism.

Proof. Put $\mu=1$ in (3.16). By the same reasoning as in the proof of Theorem 2.3, there exists a unique generalized Euler-Lagrange type additive mapping $H: A \rightarrow B$ defined by

$$
H(x)=\lim _{k \rightarrow \infty} \frac{f\left(2^{k} x\right)}{2^{k}}
$$

for all $x \in A$. By the same reasoning as in the proof of [4], the generalized Euler-Lagrange type additive mapping $H: A \rightarrow B$ is $\mathbb{R}$-linear.

By the same method as in the proof of Theorem 2.4, we have

$$
\begin{gathered}
\left\|D_{\mu, r_{1}, \ldots, r_{n}} H(0, \ldots, 0, \underbrace{x}_{j \text { th }}, 0, \ldots, 0)\right\|_{Y} \\
\quad=\lim _{k \rightarrow \infty} \frac{1}{2^{k}}\left\|D_{\mu, r_{1}, \ldots, r_{n}} f(0, \ldots, 0, \underbrace{2^{k} x}_{j \text { th }}, 0, \ldots, 0)\right\|_{Y} \\
\quad \leq \lim _{k \rightarrow \infty} \frac{1}{2^{k}} \varphi(0, \ldots, 0, \underbrace{2^{k} x}_{j \text { th }}, 0, \ldots, 0)=0
\end{gathered}
$$

for all $x \in A$. So

$$
r_{j} \mu H(x)=H\left(r_{j} \mu x\right)
$$

for all $x \in A$. Since $H\left(r_{j} x\right)=r_{j} H(x)$ for all $x \in X$ and $r_{j} \neq 0$,

$$
H(\mu x)=\mu H(x)
$$

for $\mu=i, 1$ and for all $x \in A$.

For each element $\lambda \in \mathbb{C}$ we have $\lambda=s+i t$, where $s, t \in \mathbb{R}$. Thus

$$
\begin{aligned}
H(\lambda x) & =H(s x+i t x)=s H(x)+t H(i x) \\
& =s H(x)+i t H(x)=(s+i t) H(x)=\lambda H(x)
\end{aligned}
$$

for all $\lambda \in \mathbb{C}$ and all $x \in A$. So

$$
H(\zeta x+\eta y)=H(\zeta x)+H(\eta y)=\zeta H(x)+\eta H(y)
$$


for all $\zeta, \eta \in \mathbb{C}$ and all $x, y \in A$. Hence the generalized Euler-Lagrange type additive mapping $H: A \rightarrow B$ is $\mathbb{C}$-linear. The rest of the proof is the same as in the proof of Theorem 3.5.

The following theorem is an alternative result of Theorem 3.9.

Theorem 3.10. Let $f: A \rightarrow B$ be a mapping with $f(0)=0$ for which there is a function $\phi: A^{n} \rightarrow$ $[0, \infty)$ satisfying $(2.36),(2.37),(3.7)$ and

$$
\left\|D_{\mu, r_{1}, \ldots, r_{n}} f\left(x_{1}, \ldots, x_{n}\right)\right\|_{B} \leq \phi\left(x_{1}, \ldots, x_{n}\right),
$$

for $\mu=i, 1$ and all $x, x_{1}, \ldots, x_{n} \in A$. Assume that $\lim _{k \rightarrow \infty} 2^{k} f\left(e / 2^{k}\right)$ is invertible and for each fixed $x \in A$ the mapping $t \mapsto f(t x)$ is continuous in $t \in \mathbb{R}$. Then the mapping $f: A \rightarrow B$ is a $C^{*}$-algebra homomorphism.

Remark 3.11. In Theorem 3.10, one can assume that $\sum_{k=1}^{n} r_{k} \neq 0$ instead of $f(0)=0$.

\section{Acknowledgments}

The authors would like to thank the referees for a number of valuable suggestions regarding a previous version of this paper. C. Park author was supported by Basic Science Research Program through the National Research Foundation of Korea funded by the Ministry of Education, Science and Technology (NRF-2009-0070788).

\section{References}

[1] S. M. Ulam, A Collection of Mathematical Problems, Interscience Tracts in Pure and Applied Mathematics, no. 8, Interscience, New York, NY, USA, 1960.

[2] D. H. Hyers, "On the stability of the linear functional equation," Proceedings of the National Academy of Sciences of the United States of America, vol. 27, pp. 222-224, 1941.

[3] T. Aoki, "On the stability of the linear transformation in Banach spaces," Journal of the Mathematical Society of Japan, vol. 2, pp. 64-66, 1950.

[4] Th. M. Rassias, "On the stability of the linear mapping in Banach spaces," Proceedings of the American Mathematical Society, vol. 72, no. 2, pp. 297-300, 1978.

[5] J. M. Rassias, "On approximation of approximately linear mappings by linear mappings," Journal of Functional Analysis, vol. 46, no. 1, pp. 126-130, 1982.

[6] J. M. Rassias, "On approximation of approximately linear mappings by linear mappings," Bulletin des Sciences Mathématiques, vol. 108, no. 4, pp. 445-446, 1984.

[7] J. M. Rassias, "Solution of a problem of Ulam," Journal of Approximation Theory, vol. 57, no. 3, pp. 268-273, 1989.

[8] P. Găvruţa, "A generalization of the Hyers-Ulam-Rassias stability of approximately additive mappings," Journal of Mathematical Analysis and Applications, vol. 184, no. 3, pp. 431-436, 1994.

[9] F. Skof, "Local properties and approximation of operators," Rendiconti del Seminario Matematico e Fisico di Milano, vol. 53, pp. 113-129, 1983.

[10] P. W. Cholewa, "Remarks on the stability of functional equations," Aequationes Mathematicae, vol. 27, no. 1-2, pp. 76-86, 1984.

[11] St. Czerwik, "On the stability of the quadratic mapping in normed spaces," Abhandlungen aus dem Mathematischen Seminar der Universität Hamburg, vol. 62, pp. 59-64, 1992.

[12] J. M. Rassias, "On the stability of the Euler-Lagrange functional equation," Chinese Journal of Mathematics, vol. 20, no. 2, pp. 185-190, 1992.

[13] J. M. Rassias, "On the stability of the non-linear Euler-Lagrange functional equation in real normed linear spaces," Journal of Mathematical and Physical Sciences, vol. 28, no. 5, pp. 231-235, 1994. 
[14] A. Grabiec, "The generalized Hyers-Ulam stability of a class of functional equations," Publicationes Mathematicae Debrecen, vol. 48, no. 3-4, pp. 217-235, 1996.

[15] J. M. Rassias, "On the stability of the general Euler-Lagrange functional equation," Demonstratio Mathematica, vol. 29, no. 4, pp. 755-766, 1996.

[16] M. Amyari, C. Baak, and M. S. Moslehian, "Nearly ternary derivations," Taiwanese Journal of Mathematics, vol. 11, no. 5, pp. 1417-1424, 2007.

[17] C.-Y. Chou and J.-H. Tzeng, “On approximate isomorphisms between Banach *-algebras or C*algebras," Taiwanese Journal of Mathematics, vol. 10, no. 1, pp. 219-231, 2006.

[18] M. Eshaghi Gordji, J. M. Rassias, and N. Ghobadipour, "Generalized Hyers-Ulam stability of generalized (N,K)-derivations," Abstract and Applied Analysis, vol. 2009, Article ID 437931, 8 pages, 2009.

[19] M. Eshaghi Gordji, T. Karimi, and S. Kaboli Gharetapeh, “Approximately $n$-Jordan homomorphisms on Banach algebras," Journal of Inequalities and Applications, vol. 2009, Article ID 870843, 8 pages, 2009.

[20] Z.-X. Gao, H.-X. Cao, W.-T. Zheng, and L. Xu, "Generalized Hyers-Ulam-Rassias stability of functional inequalities and functional equations," Journal of Mathematical Inequalities, vol. 3, no. 1, pp. 63-77, 2009.

[21] P. Găvruța, "On the stability of some functional equations," in Stability of Mappings of Hyers-Ulam Type, Hadronic Press Collection of Original Articles, pp. 93-98, Hadronic Press, Palm Harbor, Fla, USA, 1994.

[22] P. Găvruța, “On a problem of G. Isac and Th. M. Rassias concerning the stability of mappings,” Journal of Mathematical Analysis and Applications, vol. 261, no. 2, pp. 543-553, 2001.

[23] P. Găvruţa, "On the Hyers-Ulam-Rassias stability of the quadratic mappings," Nonlinear Functional Analysis and Applications, vol. 9, no. 3, pp. 415-428, 2004.

[24] D. H. Hyers, G. Isac, and Th. M. Rassias, Stability of Functional Equations in Several Variables, Progress in Nonlinear Differential Equations and Their Applications, no. 34, Birkhäuser, Boston, Mass, USA, 1998.

[25] D. H. Hyers, G. Isac, and Th. M. Rassias, "On the asymptoticity aspect of Hyers-Ulam stability of mappings," Proceedings of the American Mathematical Society, vol. 126, no. 2, pp. 425-430, 1998.

[26] K.-W. Jun and H.-M. Kim, "On the Hyers-Ulam stability of a difference equation," Journal of Computational Analysis and Applications, vol. 7, no. 4, pp. 397-407, 2005.

[27] K.-W. Jun and H.-M. Kim, "Stability problem of Ulam for generalized forms of Cauchy functional equation," Journal of Mathematical Analysis and Applications, vol. 312, no. 2, pp. 535-547, 2005.

[28] K.-W. Jun and H.-M. Kim, "Stability problem for Jensen-type functional equations of cubic mappings," Acta Mathematica Sinica, vol. 22, no. 6, pp. 1781-1788, 2006.

[29] K.-W. Jun and H.-M. Kim, "Ulam stability problem for a mixed type of cubic and additive functional equation," Bulletin of the Belgian Mathematical Society. Simon Stevin, vol. 13, no. 2, pp. 271-285, 2006.

[30] K.-W. Jun, H.-M. Kim, and J. M. Rassias, "Extended Hyers-Ulam stability for Cauchy-Jensen mappings," Journal of Difference Equations and Applications, vol. 13, no. 12, pp. 1139-1153, 2007.

[31] A. Najati, "Hyers-Ulam stability of an n-Apollonius type quadratic mapping," Bulletin of the Belgian Mathematical Society. Simon Stevin, vol. 14, no. 4, pp. 755-774, 2007.

[32] A. Najati, "On the stability of a quartic functional equation," Journal of Mathematical Analysis and Applications, vol. 340, no. 1, pp. 569-574, 2008.

[33] A. Najati and M. B. Moghimi, "Stability of a functional equation deriving from quadratic and additive functions in quasi-Banach spaces," Journal of Mathematical Analysis and Applications, vol. 337, no. 1, pp. 399-415, 2008.

[34] A. Najati and C. Park, "Hyers-Ulam-Rassias stability of homomorphisms in quasi-Banach algebras associated to the Pexiderized Cauchy functional equation," Journal of Mathematical Analysis and Applications, vol. 335, no. 2, pp. 763-778, 2007.

[35] A. Najati and C. Park, "On the stability of an $n$-dimensional functional equation originating from quadratic forms," Taiwanese Journal of Mathematics, vol. 12, no. 7, pp. 1609-1624, 2008.

[36] A. Najati and C. Park, "The Pexiderized Apollonius-Jensen type additive mapping and isomorphisms between $C^{*}$-algebras," Journal of Difference Equations and Applications, vol. 14, no. 5, pp. 459-479, 2008.

[37] C.-G. Park, "On the stability of the linear mapping in Banach modules," Journal of Mathematical Analysis and Applications, vol. 275, no. 2, pp. 711-720, 2002.

[38] C.-G. Park, "Linear functional equations in Banach modules over a C*-algebra," Acta Applicandae Mathematicae, vol. 77, no. 2, pp. 125-161, 2003.

[39] C. G. Park, “Universal Jensen's equations in Banach modules over a $C^{*}$-algebra and its unitary group," Acta Mathematica Sinica, vol. 20, no. 6, pp. 1047-1056, 2004. 
[40] C.-G. Park, "Lie *-homomorphisms between Lie $C^{*}$-algebras and Lie $*$-derivations on Lie $C^{*}$ algebras," Journal of Mathematical Analysis and Applications, vol. 293, no. 2, pp. 419-434, 2004.

[41] C.-G. Park, "Homomorphisms between Lie JC*-algebras and Cauchy-Rassias stability of Lie JC*algebra derivations," Journal of Lie Theory, vol. 15, no. 2, pp. 393-414, 2005.

[42] C.-G. Park, "Generalized Hyers-Ulam-Rassias stability of $n$-sesquilinear-quadratic mappings on Banach modules over $C^{*}$-algebras," Journal of Computational and Applied Mathematics, vol. 180, no. 2, pp. 279-291, 2005.

[43] C.-G. Park, "Homomorphisms between Poisson $J C^{*}$-algebras," Bulletin of the Brazilian Mathematical Society, vol. 36, no. 1, pp. 79-97, 2005.

[44] C.-G. Park and J. Hou, "Homomorphisms between $C^{*}$-algebras associated with the Trif functional equation and linear derivations on $C^{*}$-algebras," Journal of the Korean Mathematical Society, vol. 41, no. 3, pp. 461-477, 2004.

[45] C. Park and J. M. Park, "Generalized Hyers-Ulam stability of an Euler-Lagrange type additive mapping," Journal of Difference Equations and Applications, vol. 12, no. 12, pp. 1277-1288, 2006.

[46] J. M. Rassias and M. J. Rassias, "Asymptotic behavior of alternative Jensen and Jensen type functional equations," Bulletin des Sciences Mathématiques, vol. 129, no. 7, pp. 545-558, 2005.

[47] M. J. Rassias and J. M. Rassias, "Refined Hyers-Ulam superstability of approximately additive mappings," Journal of Nonlinear Functional Analysis and Differential Equations, vol. 1, no. 2, pp. 175$182,2007$.

[48] J. M. Rassias, "Solution of the Ulam stability problem for Euler-Lagrange quadratic mappings," Journal of Mathematical Analysis and Applications, vol. 220, no. 2, pp. 613-639, 1998.

[49] Th. M. Rassias, "On the stability of the quadratic functional equation and its applications," Universitatis Babeş-Bolyai. Studia. Mathematica, vol. 43, no. 3, pp. 89-124, 1998.

[50] Th. M. Rassias, "The problem of S. M. Ulam for approximately multiplicative mappings," Journal of Mathematical Analysis and Applications, vol. 246, no. 2, pp. 352-378, 2000.

[51] Th. M. Rassias, "On the stability of functional equations in Banach spaces," Journal of Mathematical Analysis and Applications, vol. 251, no. 1, pp. 264-284, 2000.

[52] Th. M. Rassias, "On the stability of functional equations and a problem of Ulam," Acta Applicandae Mathematicae, vol. 62, no. 1, pp. 23-130, 2000.

[53] Th. M. Rassias and P. Šemrl, "On the Hyers-Ulam stability of linear mappings," Journal of Mathematical Analysis and Applications, vol. 173, no. 2, pp. 325-338, 1993.

[54] Th. M. Rassias and K. Shibata, "Variational problem of some quadratic functionals in complex analysis," Journal of Mathematical Analysis and Applications, vol. 228, no. 1, pp. 234-253, 1998.

[55] D. Zhang and H.-X. Cao, "Stability of group and ring homomorphisms," Mathematical Inequalities $\mathcal{E}$ Applications, vol. 9, no. 3, pp. 521-528, 2006.

[56] Z. Gajda, "On stability of additive mappings," International Journal of Mathematics and Mathematical Sciences, vol. 14, no. 3, pp. 431-434, 1991.

[57] R. V. Kadison and J. R. Ringrose, Fundamentals of the Theory of Operator Algebras. Vol. I, vol. 100 of Pure and Applied Mathematics, Academic Press, New York, NY, USA, 1983. 